\title{
IShTAR ICRF antenna field characterization in vacuum and plasma by using probe diagnostic
}

\author{
Mariia Usoltceva ${ }^{1,2,3,{ }^{*}}$, Roman Ochoukov ${ }^{2}$, Rodolphe D’Inca ${ }^{2}$, Jonathan Jacquot ${ }^{2}$, Kristel Crombé ${ }^{1,4}$, Ana Kostic $^{1,2}$, \\ Stéphane Heuraux ${ }^{3}$, Eric Faudot ${ }^{3}$, Jean-Marie Noterdaeme ${ }^{1,2}$, and the IShTAR team ${ }^{2}$ \\ ${ }^{1}$ Department of Applied Physics, Gent University, 9000 Gent, Belgium \\ ${ }^{2}$ Max-Planck-Institut für Plasmaphysik, Boltzmannstr. 2, 85748 Garching, Germany \\ ${ }^{3}$ Université de Lorraine, 54506 Vandœuvre-lès-Nancy, France \\ ${ }^{4}$ LPP-ERM-KMS, TEC partner, 1000 Brussels, Belgium
}

\begin{abstract}
RF sheath physics is one of the key topics relevant for improvements of ICRF heating systems, which are present on nearly all modern magnetic fusion machines. This paper introduces developement and validation of a new approach to understanding general RF sheath physics. The presumed reason of enhanced plasma-antenna interactions, parallel electric field, is not measured directly, but proposed to be obtained from simulations in COMSOL Multiphysics ${ }^{\circledR}$ Modeling Software. Measurements of RF magnetic field components with B-dot probes are done on a linear device IShTAR (Ion cyclotron Sheath Test ARrangement) and then compared to simulations. Good resulting accordance is suggested to be the criterion for trustworthiness of parallel electric field estimation as a component of electromagnetic field in modeling. A comparison between simulation and experiment for one magnetic field component in vacuum has demonstrated a close match. An additional complication to this ICRF antenna field characterization study is imposed by the helicon antenna which is used as a plasma ignition tool in the test arrangement. The plasma case, in contrast to the vacuum case, must be approached carefully, since the overlapping of ICRF antenna and helicon antenna fields occurs. Distinguishing of the two fields is done by an analysis of correlation between measurements with both antennas together and with each one separately.
\end{abstract}

\section{Introduction}

Interaction between ICRF antenna fields and plasma of a tokamak edge has been studied for decades. RF sheathaccelerated ions have been shown to be the main reason of antenna limiters sputtering [1]. Attempts done on different experimental machines to reduce negative effects accompanying plasma-wall interactions are usually heuristic, with conclusions drawn basing on consequential parameters like impurities concentration, $\mathrm{RF}$ currents or temperature of limiting structures $[2,3]$, not on the electrical field. Improvements are typically achieved for specific machine parameters, magnetic field angle, antenna geometry, toroidal phasing, etc. Beside that, modelling tools have been developed for SOL ICRF physics simulations. They are reviewed in details in [4].

In this paper, an approach to understand general RF sheath physics is described and first results are presented. Parallel electric field, supposedly responsible for the enhancement of interactions between antenna and plasma, is inherently connected to the magnetic field of an RF wave. Experimental results of magnetic field components measurements in the linear device IShTAR are used as a reference for simulations in COMSOL Multiphysics ${ }^{\circledR}$ Modeling Software in order to obtain target parallel electric field values.

\section{Field characterization with probe diagnostic}

The object of interest, a thin layer in the vicinity of an antenna, is hardly approachable by diagnostic tools, especially since the most common (and so far the only one applicable in SOL plasma) diagnostic for potential measurements, electrical probes, is known to introduce significant changes by its presence. That is why indirect measurements are preferred, though their results should be necessarily linked to the E-field values.

The electromagnetic field of an ICRF antenna can be simulated and values for each component of the field can be calculated separately. A relatively simple B-dot probe diagnostic is used for magnetic field measurements in our experimental device. A B-dot probe is able to provide local measurement of one component of the magnetic field. Assuming that agreement of experimental and numerical results for magnetic field components would mean the equality of electrical field values, we can expect to deduce the sought parallel electric field from simulation, as soon as we confirm the match of experiment and simulation results for the magnetic field. 


\section{Experimental setup}

The objectives of IShTAR (Ion cyclotron Sheath Test ARrangement) are described in [5,6]. Fig. 1 illustrates all important features of the device: main vacuum chamber of 0.5 meter radius with an ICRF antenna (5.22 MHz, 1 $\mathrm{kW}$ of power) and a manipulator in front of it; small vacuum chamber attached on a side, with helicon antenna for plasma ignition (11.76 MHz, up to $3 \mathrm{~kW}$ ); two sets of magnetic coils of up to $0.24 \mathrm{~T}$ and $0.03 \mathrm{~T}$, surrounding correspondingly big and small vacuum chambers. This setup allows around 10 second of plasma confinement, with a high density $\left(\sim 10^{17} \mathrm{~m}^{-3}\right)$ plasma column of $15-20 \mathrm{~cm}$ in radius being in close proximity to the specially designed one-strap ICRF antenna. The working gas is $\mathrm{He}$ (Ar can be also used).

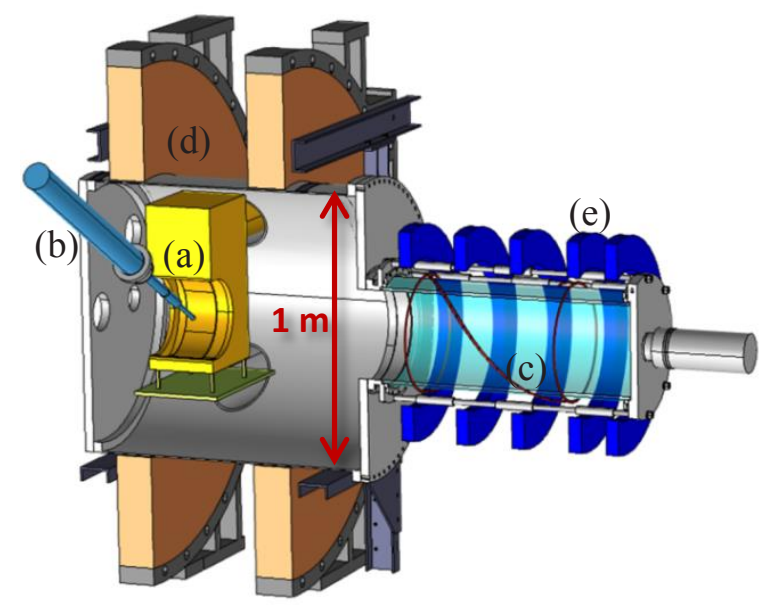

Fig. 1. IShTAR scheme: (a) ICRF antenna, (b) manipulator, (c) helicon antenna, (d) big magnetic coils, (e) small magnetic coils.

ICRF antenna magnetic field components are measured by B-dot probes. An array of 4 probes (Fig. 2) is installed on a radially movable manipulator in parallel to the main magnetic field (z-direction) with coordinates of $-15,-5,5$ and $15 \mathrm{~cm}$ counting from the ICRF antenna center.

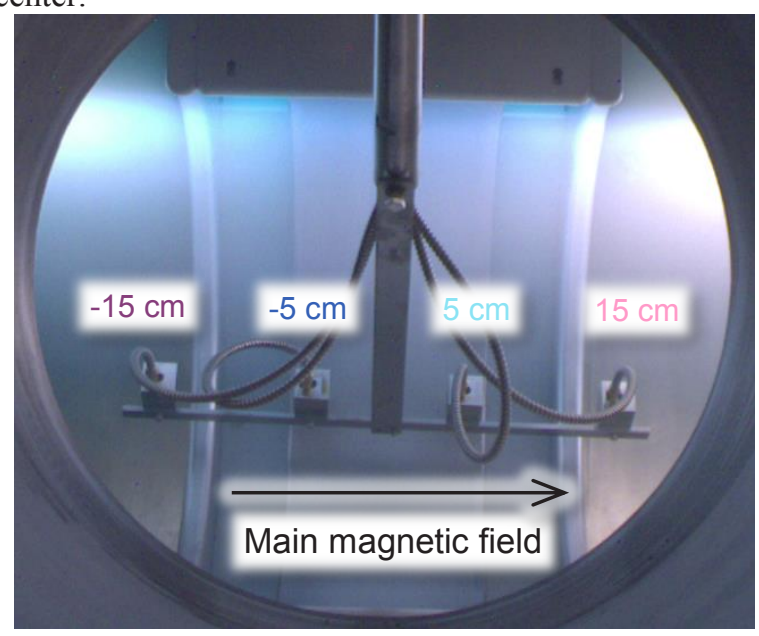

Fig. 2. Probe array on a manipulator in front of an ICRF antenna inside IShTAR.

\section{Experimental results}

The first thing that should be noted before proceeding to the experimental results, is that there is more than one radio-frequency antenna in the experimental setup. The helicon antenna situated in the small chamber and dedicated to start a discharge also emits RF waves. Its presence complicates the task of ICRF antenna magnetic field measurements and its contribution must always be taken into account.

In order to distinguish the magnetic fields of the two antennas, following scenarios have been studied:

(a) Plasma with only helicon antenna.

(b) Plasma with only ICRF antenna (since this antenna is not designed to ignite plasma, only weak plasma is produced; no wave propagation is registered, evanescent wave signal decays as in vacuum).

(c) Plasma with both antennas simultaneously.
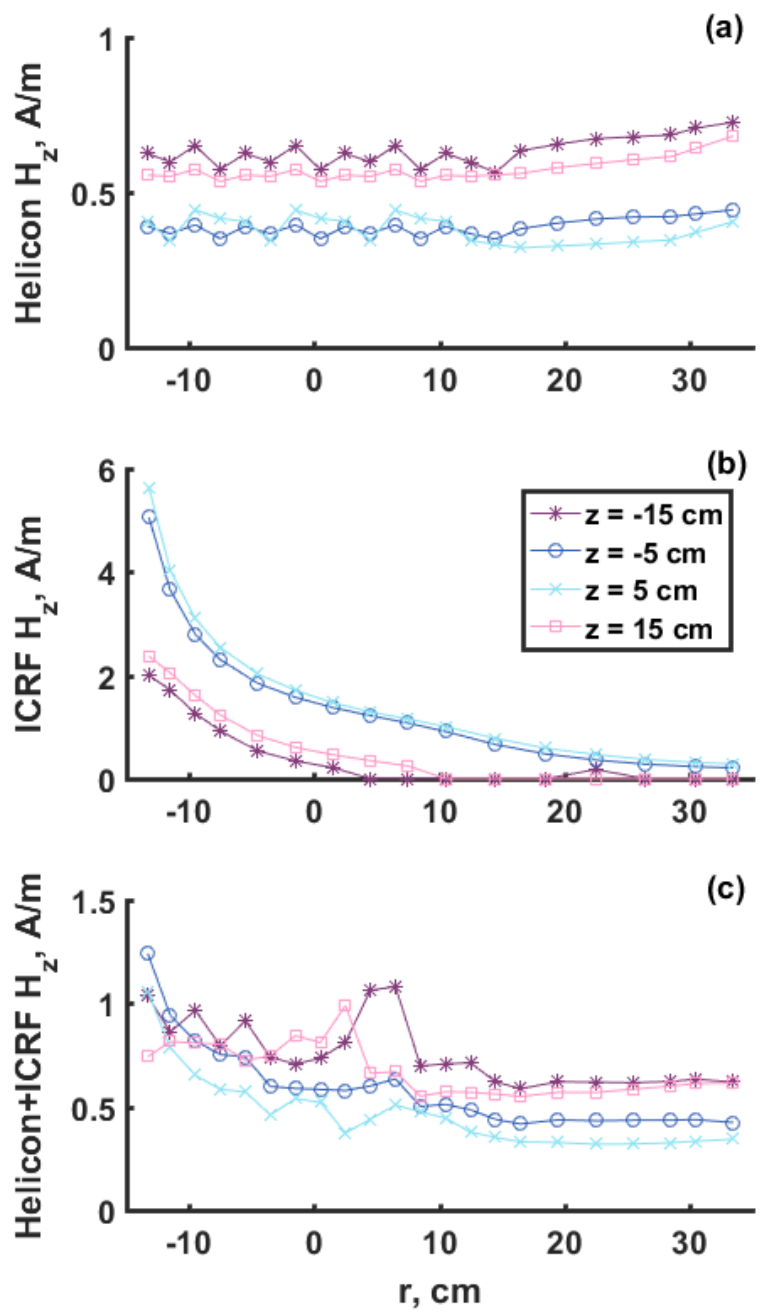

Fig. 3. Experimental $\mathrm{H}_{\mathrm{z}}(\mathrm{r}$ ) of: (a) helicon antenna, (b) ICRF antenna, (c) helicon and ICRF antennas together.

Experimental results (Fig. 3) reveal evident superposition of the two antenna fields. In the range of positive values of radius (farther away from the ICRF antenna) profiles of $\mathrm{H}_{\mathrm{z}}$ for all 4 probes have similar shape on two plots: Fig. 3a which shows the case with helicon antenna only, and Fig. 3c which is for the case with two antennas. In the vicinity of the ICRF antenna 
(negative r) measurements demonstrate the impact of the ICRF antenna field on the resulting signal. A significant rise of signal towards the ICRF antenna, similar in shape to that observed on Fig. 3b, is especially well seen for the two central probes.

It can be noticed, however, that absolute values of ICRF antenna magnetic field on Fig $3 b$ are significantly higher than those measured in plasma with both antennas. The reasons is the B-dot probes detectors, which have a constant attenuation factor for frequencies greater than $10 \mathrm{MHz}$, thus aiming to cut low-frequency noise. It is still possible to measure the $5.22 \mathrm{MHz}$ signal of ICRF antenna with those detectors, using a different calibration (as it was done for results on Fig. 3b). But in combination with the $11,76 \mathrm{MHz}$ frequency of the helicon antenna the signal of ICRF antenna becomes weak, being attenuated much stronger than the helicon antenna signal. For a complete analysis of the superimposed magnetic fields of the two antennas new detectors need to be made, with equal attenuation factor for both frequencies. Nevertheless, it can be already concluded that it is feasible to distinguish the ICRF antenna magnetic field from the sum of the two fields.

\section{Simulation}

Realistic geometry of all in-vessel parts (including ICRF antenna, manipulator and probes) has been built in COMSOL Multiphysics ${ }^{\circledR}$. Fig. 4 is a view on the ICRF antenna without a side wall: the antenna strap, the limiting (supporting) structure and the feeding coaxial transmission line can be seen. On the background the small chamber is visible.

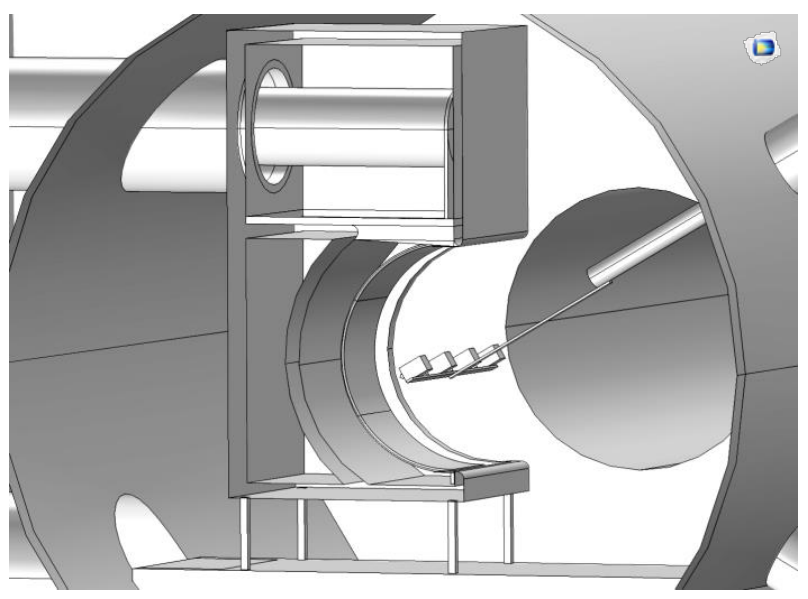

Fig. 4. IShTAR model with all main components: ICRF antenna (side wall removed), manipulator and probes.

\subsection{Vacuum case}

The standard Comsol Electromagnetics Module makes it possible to model the vacuum electromagnetic field of the IShTAR ICRF antenna. In vacuum, an RF wave of $5.22 \mathrm{MHz}$ propagates in the coaxial transmission line and then becomes evanescent, causing very small fields inside the IShTAR chamber. Magnetic field distribution in logarithmic scale $(\log$ of $\mathrm{A} / \mathrm{m})$ is plotted on Fig. 5.

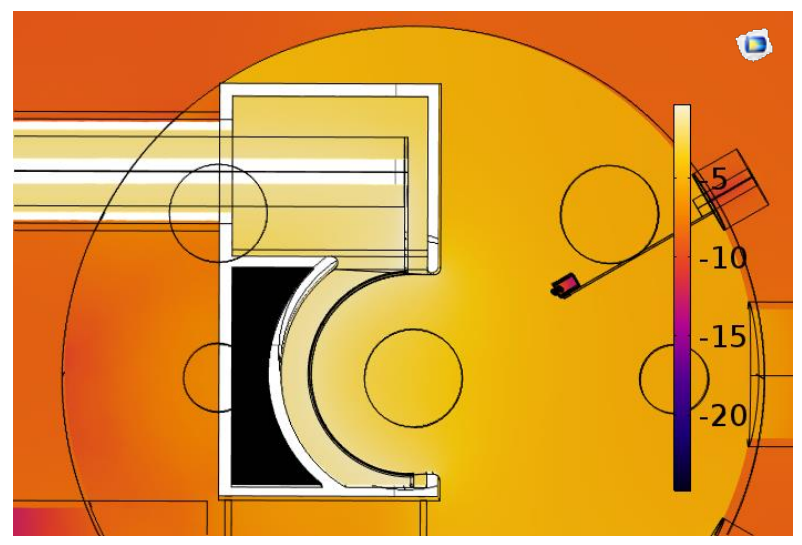

Fig. 5. Magnetic field of IShTAR ICRF antenna in vacuum in logarithmic scale.

Magnetic field measured by a B-dot probe is represented in simulation as an average field inside a cylindrical volume at the position and of the size of a real inductor used in a probe. Radial profiles of $\mathrm{H}_{\mathrm{z}}$ at $5.22 \mathrm{MHz}$ for the 4 probes (Fig. 6) have the same shape in the experiment and in the modeling. Due to a perfect symmetry of the ICRF antenna and probes in the model results for two outer and two central probes are identical, which is well reproduced in the experiment. Absolute values do not play any role here, since they are fully dependent on the amount of injected power and the matching system parameters. This can be always varied in the simulation, if needed.
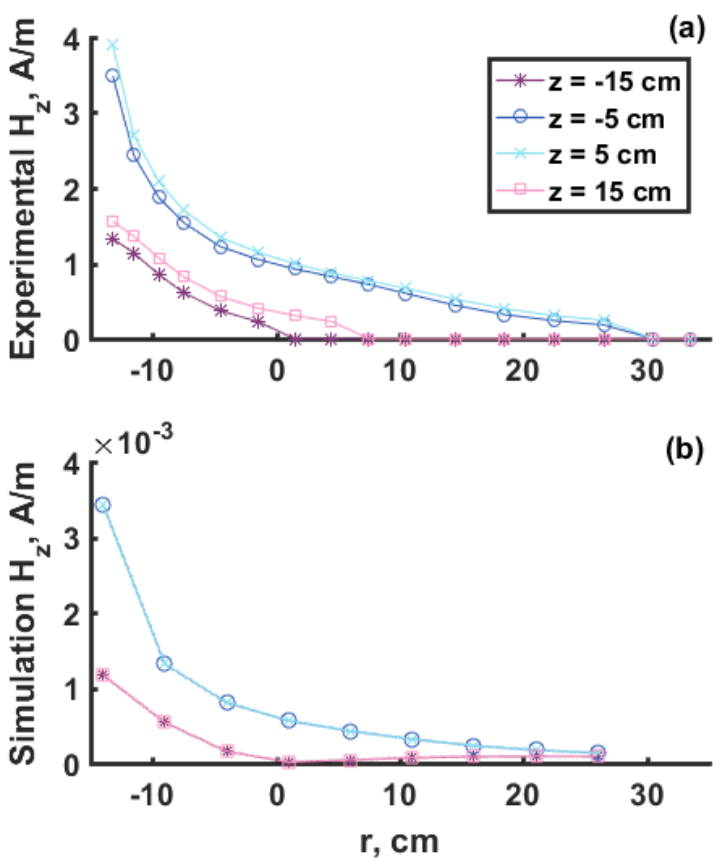

Fig. 6. $H_{z}(r)$ in vacuum: (a) measured in experiment, (b) calculated in simulation.

Four radial profiles obviously do not provide a full $2 \mathrm{D}$ plot of the magnetic field parameters. Here we are making an assumption that if a comparison of 4 radial experimental and simulation profiles is successful, an electromagnetic field distribution from the simulation is considered to closely depict the real field in our device. 
For more certainty not only one component of magnetic field can be compared, but at least 2 .

Since a simulation of electromagnetic field in COMSOL gives all components of the field at any geometrical point, parallel electric field is thus known at any required position. Calculated $\mathrm{H}_{\mathrm{z}}$ and $\mathrm{E}_{\mathrm{z}}$ in front of the antenna are provided as examples in Fig. 7 and 8.

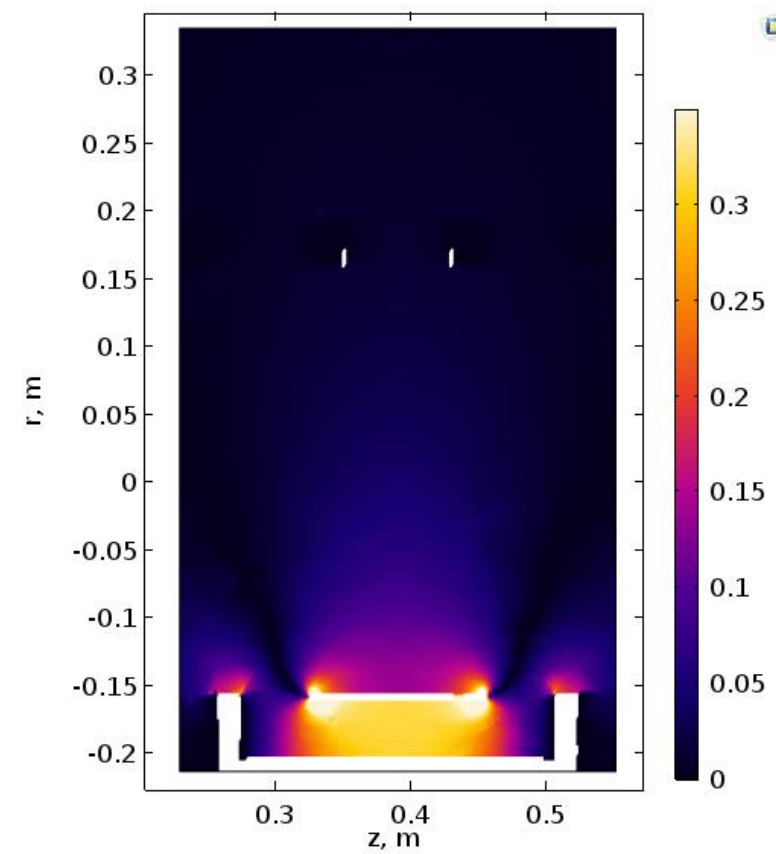

Fig. 7. Calculated 2D distribution of $\mathrm{H}_{\mathrm{z}}$ (in $\mathrm{A} / \mathrm{m}$ ) in front of the ICRF antenna in vacuum.

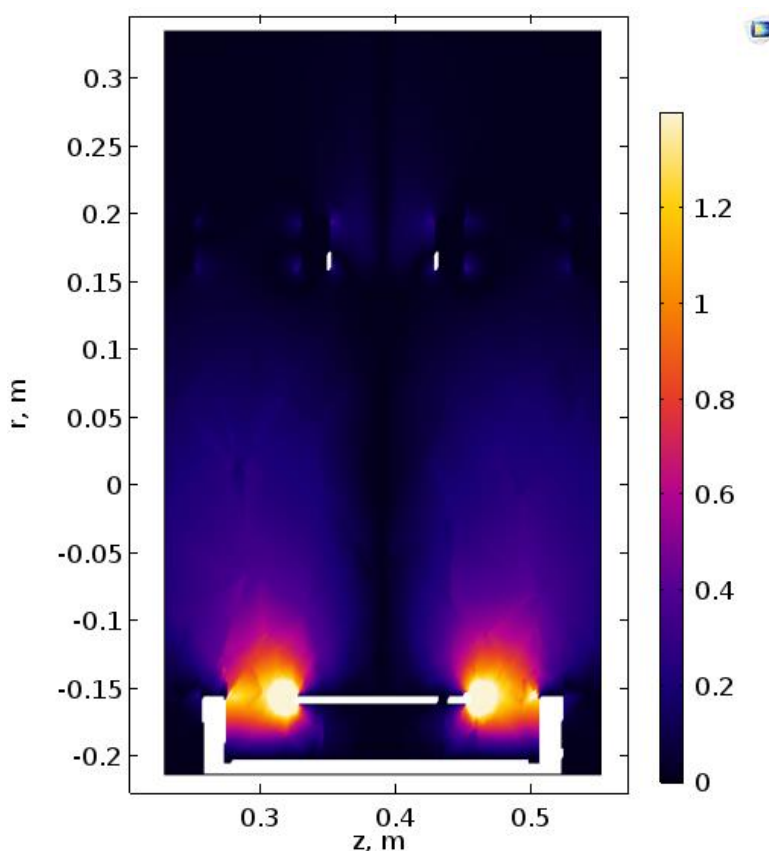

Fig. 8. Calculated $2 \mathrm{D}$ distribution of $\mathrm{E}_{\mathrm{z}}($ in $\mathrm{V} / \mathrm{m}$ ) in front of the ICRF antenna in vacuum.

\subsection{Plasma case}

Plasma can be programmed in COMSOL's Electromagnetics Module as a material with manually assigned physical properties. The next step of the validation of the approach presented in this paper is planned to be a plasma simulation in IShTAR geometry. Ideally, each case (ICRF antenna, helicon antenna, both antennas together) needs to be addressed separately and comparisons with experiment have to be made accordingly.

\section{Conclusion}

An approach for obtaining parallel electric field of an ICRF antenna using a combination of experiment and simulation is presented. The obtained agreement of experimental and modelling results for one of the magnetic field components in vacuum case supports the proposed assumption of feasibility of the explained approach. Further comparisons, for other magnetic field components in vacuum and for the plasma case will provide more confident and detailed summary of the applicability of the discussed approach.

This work has been carried out within the framework of the EUROfusion Consortium and has received funding from the Euratom research and training programme 2014-2018 under grant agreement No 633053. The views and opinions expressed herein do not necessarily reflect those of the European Commission.

\section{References}

1. D.A. D'Ippolito and J.R. Myra, Plasma Phys. Control. Fusion 33, 607 (1991)

2. V.Bobkov et al., Nucl. Mater. Energy (2016)

3. W. Zhang et al., 22nd RFPPC (2017)

4. L. Colas et al., 22nd RFPPC (2017)

5. K. Crombé et al., 26th IAEA Fusion Energy Conference (2016)

6. R. D'Inca et al., AIP Conference (2015) 